
\title{
25 Research Square \\ SVM Classification of Brain Gray Matter Volume Predicts Classic Trigeminal Neuralgia
}

\section{Xinji Peng}

First Affiliated Hospital of Nanchang University https://orcid.org/0000-0002-4235-6203

\section{You Chen}

Nanchang University Second Affiliated Hospital

\section{Guoshu Zhao}

First Affiliated Hospital of Nanchang University

\section{Huifang Du}

First Affiliated Hospital of Nanchang University

\section{Ziyi Hu}

First Affiliated Hospital of Nanchang University

Laichang He ( $\square$ laichang_he@163.com)

First Affiliated Hospital of Nanchang University https://orcid.org/0000-0001-7277-2104

\section{Research article}

Keywords: SVM, gray matter volume, classical trigeminal neuralgia, classification

Posted Date: May 24th, 2021

DOI: https://doi.org/10.21203/rs.3.rs-551229/v1

License: (c) (i) This work is licensed under a Creative Commons Attribution 4.0 International License. Read Full License 


\section{Abstract}

Background: Previous studies have shown gray matter(GM) abnormalities in the central nervous system at the group level, but this method is limited because it is based on single or cluster voxels. In contrast, machine learning makes full use of all available empirical information, including differences in brain images or behavioral data, to classify or predict data and ensure good generalization ability. This approach has potential use as a prediction tool at the individual level. We thus hypothesized that a multivariate pattern classification method may distinguish classic trigeminal neuralgia(CTN) patients from healthy controls $(\mathrm{HC})$ based on gray matter volume(GMV).

Methods: Resting-state fMRI scans in $24 \mathrm{CTN}$ and $22 \mathrm{HC}$ were processed to extract whole-brain GMV. Based on this feature dataset, take the method of Spearman, T-test, F-score, principal component analysis(PCA) respectively to reduce the feature dimension. Applying a linear support vector machine(SVM) algorithm to differentiate CTN and HC. And extract the features that survive each iteration. Pearson correlation analysis was then used to assess the correlations between the deci_value(the distance from the sample to the optimal hyperplane) and VAS scores and pain duration respectively in CTN patients.

Results: Compared with other methods of feature dimension reduction, PCA has a higher ability to correctly classify an individual with an accuracy of $85 \%$ (AUC $0.9223 ; 91.67 \%$ sensitivity; $86.36 \%$ specificity, $p<0.001)$. And the features that survive each iteration were concentrated in the region of the left anterior cingulate cortex(ACC_L), right superior frontal gyrus(SFG_R), and bilateral cerebellum inferior $(\mathrm{Cl})$. In the CTN group, deci_value was positively correlated with the VAS scores in PCA $(r=0.42$, $\mathrm{P}=0.041$, two-tailed). While there was no difference between deci_value and VAS scores in F-score, T-test, Spearman $(r=0.39, p=0.06 / r=0.36, p=0.09 / r=0.37, p=0.08$ respectively $)$. Also, we did not find significant correlations between the pain duration for CTN patients and deci_value among the four methods of feature dimension reduction $(P>0.05)$.

Conclusions: This study demonstrates that machine-learning algorithms can detect characteristic patterns of structural alterations in TN, and not only highlights the high accuracy of the PCA method, but also the role of ACC, SFG, and $\mathrm{Cl}$ for classification.

\section{Background}

According to incomplete statistics, the incidence of trigeminal neuralgia (TN) is about 52.2/100000 in China, and classical trigeminal neuralgia (CTN) accounts for the vast majority. Vascular compression of the trigeminal nerve can cause unbearable pulse pain ${ }^{[1]}$, and long-term pain stimulation not only seriously affects the quality of patients' life, but also brings a heavy medical burden to the country and society ${ }^{[2,3]}$. The clearest source of CTN is widely considered to be neurovascular compression of the trigeminal nerve at the root entry zone at the brainstem ${ }^{[4]}$. Previous studies have shown gray matter(GM) and white matter(WM) abnormalities in the central nervous system at the group level ${ }^{[5-7]}$, but this method is limited 
because it is based on single or cluster voxels. In contrast, machine learning ${ }^{[5-7]}$ makes full use of all available empirical information, including differences in brain images or behavioral data, to classify or predict data and ensure good generalization ability. And support vector machine (SVM)-based algorithm ${ }^{[10]}$ has been successfully used to classify chronic pain using voxel-based gray matter volume $(\mathrm{GMV})^{[10]}$. We thus hypothesized that a multivariate pattern classification method may distinguish CTN patients from healthy controls $(\mathrm{HC})$ based on GMV.

\section{Methods}

\section{Participants}

24 CTN patients ( 9 males, 15 females; average age $55 \pm 13$ years; average duration $3.4 \pm 4.2$ years; average VAS $6.1 \pm 1.4$ ) and $22 \mathrm{HC}$ (13 males, 9 females; average age $55 \pm 11$ years) were included in this study. Inclusion criteria: reference to $(\mathrm{ICDH}-3)^{[13]}$. Exclusion criteria: I) other types of chronic pain conditions, II) history of other central nervous system diseases or mental illness, III) other somatic or psychiatric conditions, IV) unsuitable for magnetic resonance scanning. Demographics and behavioral results of CTN and $\mathrm{HC}$ groups were listed in Table 1. And there is no significant difference between CTN and $\mathrm{HC}$ groups in age, gender, and handedness. The present study was approved by the Medical Research Ethics Committee of The First Affiliated Hospital of Nanchang University. All individuals include healthy controls provided signed informed consent to participate in the study.

Table 1

Demographics and behavioral results of CTN and HC groups

\begin{tabular}{|c|c|c|c|}
\hline & CTN & $\mathrm{HC}$ & $P$ \\
\hline Gender(Male/Female) & $9 / 15$ & $13 / 9$ & 0.143 \\
\hline Age(years) & $55 \pm 13$ & $55 \pm 11$ & 0.96 \\
\hline Handedness(R/L) & $24 / 0$ & $22 / 0$ & $\otimes 0.99$ \\
\hline Duration(years) & $3.4 \pm 4.2$ & - & - \\
\hline $\operatorname{Side}(R / L)$ & $16 / 8$ & - & - \\
\hline VAS & $6.1 \pm 1.4$ & - & - \\
\hline
\end{tabular}

Independent t-tests comparing the age of the two groups. Male/female and Handedness were analyzed using a chi-squared test. CTN, classical trigeminal neuralgia; $H C$, healthy control; $R$, right; $L$, left; VAS, visual analogue scale $(P<0.05$ represented statistically significant differences)

\section{MRI parameters}


All magnetic resonance imaging (MRI) data are acquired with a 3.0T MRI scanner (Siemens AG). The total scan time was approximately $15 \mathrm{~min}$. All participants were instructed to close their eyes, keep relax, and breathe smoothly, and do not do any task or systematic thinking until the scans were completed. T1weighted MRI and resting-state functional MRI data were acquired. Parameters were as follows: 176 structural images $([T R]=1900 \mathrm{msec} ;[T E]=2.26 \mathrm{msec}$; thickness $=1 \mathrm{~mm}$; gap $=0.5 \mathrm{~mm}$; matrix size $=$ 256x256; FOV $=250 \times 250 \mathrm{~mm}$; turning angle $=90^{\circ}$; voxel size $=1 \mathrm{~mm} \times 1 \mathrm{~mm} \times 1 \mathrm{~mm}$ ) and 240 functional images $([T R]=2000 \mathrm{msec} ;[T E]=30 \mathrm{msec}$; thickness $=4 \mathrm{~mm}$; gap $=1.2 \mathrm{~mm}$; matrix size $=64 \times 64$; turning angle $=90^{\circ} ; \mathrm{FOV}=220 \times 220 \mathrm{~mm}, 29$ axial, voxel size $\left.=3 \mathrm{~mm} \times 3 \mathrm{~mm} \times 3 \mathrm{~mm}\right)$. At the same time, conventional axial T1WI, T2WI, and fluid-attenuated inversion recovery sequences were acquired for excluding intracranial lesions before the BOLD and T1 sequence. Besides, before further analysis, we carefully examined the images of each subject to ensure the quality of the data.

\section{fMRI data processing}

We applied voxel-based morphometry (VBM) to quantify GMV for individual participants, unlike traditional methods of regions of interest (ROI), VBM is not susceptible to selection bias, it can provide an unbiased method to identify brain structural abnormalities through MRI scans. I) Convert the data format of initial T1 images into an analytical image format by SPM8(http://www.fil.ion.ucl.ac.uk/spm); II)Segment the converted images into gray matter (GM), white matter(WM), and cerebrospinal fluid (CSF) with a default 0.0001 bias regularization; III) Registration of nonlinear deformations of GM images of all subjects by using the DARTEL (Diffeomorphic Anatomical Registration Through Exponentiated Lie Algebra) package (Ashburner, 2007) provided by SPM8; IV) Create a customized template by using the GM segmented images from all subjects; $V$ ) Register the individual GM images to the template and normalize to the Montreal Neurological Institute (MNI) space; vi) Be spatially smoothed with an 8mm fullwidth at half maximum (FWHM) Gaussian kernel to improve the signal-to-noise ratio of the image; VII) Extract the signal of the resulting normalized and smoothed GM images that were used for training.

\section{Statistical analysis}

Demographic and clinical variables of CTN and HC groups were statistically analyzed using SPSS 26.0 software. Independent t-tests comparing the age of the two groups. Gender and Handedness were analyzed using the chi-squared test, $P<0.05$ was considered to indicate a statistically significant difference.

\section{SVM Classification}

Machine learning in neuroimage data research can be divided into five steps ${ }^{[14]}$ : I)Feature extraction; II)Feature selection or feature dimension reduction; III)Model training and model testing; IV)Evaluation the prediction ability of the model; $\mathrm{V}$ )Feature localization that contributes to the prediction. 
To discriminate CTN from HC based on GMV, we applied an SVM-based algorithm from the Libsvm software library (https://www.csie.ntu.edu.tw/ cjlin/libsvm/) on the Matlab platform. The flow chart for SVM classification was shown in Fig. 1, which followed the same classification procedure published previously ${ }^{[15]}$. First of all, extract the signal of the 46 patients' GMV as a features dataset and normalized it to $(0,1)$. Due to the small sample size, the performance of the classifier was tested by leaving-one-out cross validation(LOOCV). The advantage of LOOCV over other cross-validation is that it can train the data as much as possible so that to obtain a more accurate classifier. Thus, within each iteration, we considered one participant as the testing dataset and the remaining participants as the training dataset. Next, Spearman, T-test, F-score, and PCA was respectively used for feature selection or feature dimension reduction to avoid overfitting and discard non-informative features. A) Spearman: Spearman correlation coefficient measures the strength of the relationship between the two variables, for each feature, it was calculated from the two groups based on GMV. B) T-test: In the process of feature selection, by calculating the test statistics, comparing the size of the statistics between features. C) F-score: Generally speaking, the larger F-score of a feature, the greater the value of this feature in classification. In the practical application of machine learning, calculating the F-score of all features. Based on the above three methods, arrange the statistical values in descending order, and then select the top $5 \%$ statistical values as features for training. D) PCA: All the features were decomposed into a series of principal components(PC), and only with a cumulative contribution of more than $90 \%$ were retained.

The misclassification parameter $\mathrm{C}$ of the SVM was optimized using nested cross-validation on the current training dataset. The process for each iteration includes finding the optimal model used to classify the test dataset. During model training, linear SVM assigns a specific weight to each feature to reflect its importance in the classification ${ }^{[16]}$. And the features that survive in each iteration were retained, this makes it possible to derive the spatial pattern underlying the classification from the mean weight across iterations for the surviving features. A positive weight indicates TN patients having higher GMV than HC in that particular region, while a negative weight indicates the opposite. After 46 iterations, the posterior balanced accuracy was calculated to evaluate the classification performance. By the way, the PCA method can not return a weight map.

Besides, to examine the degree to which the classification was driven by CTN symptoms rather than other confounds unrelated to CTN, we make a correlation between the deci_value for each subject and the VAS scores and pain duration, respectively. The method was similar to previous researches ${ }^{[17,18]}$.

Finally, the reliability of the model was evaluated by permutation test. In the permutation test, the label of the sample is randomly replaced and then repeat the above steps 1000 times. If the classifier does not acquire the corresponding relationship between the sample data and the label, then the frequency distribution of the average classification accuracy after generalization in the permutation test should obey the normal distribution with an average of $50 \%$. If the average classification accuracy after generalization based on real tags falls outside the $95 \%$ confidence interval based on random tags, it is considered that the SVM classifier does get reliable learning from training data. 


\section{Results}

\section{Overall classifier performance of different feature selection methods}

Table 2 shows the result of different feature selection methods of the SVM classification between 24 CTN and $22 \mathrm{HC}$ based on GMV. Include the overall accuracy, sensitivity, specificity, AUC, and the P_value after permutation, with Receiver Operating Characteristic(ROC) curve shown in Fig. 2A. It's not hard to see that PCA has the higher ability to correctly classify an individual as a CTN patient or HC, with $91.67 \%$ sensitivity, $86.36 \%$ specificity, and 0.9223 area under the curve (AUC), $\mathrm{P}<0.001$. Thus, $39 / 46$ subjects were correctly classified using PCA, specifically, 19/24 patients and 20/22 HC were correctly classified (Fig. 2B). And the model used the top 18 PC to explain more than $90 \%$ of the original features.

Table 2 The performance of different feature selection methods for classification

\begin{tabular}{|llllll|}
\hline & Acurracy & Sensitivity & Specificity & AUC & P \\
\hline Spearman & $73.91 \%$ & $75.00 \%$ & $77.27 \%$ & 0.7803 & 0.009 \\
\hline T-test & $71.74 \%$ & $66.67 \%$ & $81.82 \%$ & 0.7708 & 0.017 \\
\hline F-score & $71.74 \%$ & $66.67 \%$ & $81.82 \%$ & 0.7746 & 0.000 \\
\hline PCA & $84.78 \%$ & $91.67 \%$ & 86.36 & 0.9223 & 0.000 \\
\hline
\end{tabular}

\section{Relationship between deci_value and VAS scores and pain duration}

The correlation between the deci_value(the distance from the sample to the classification plane) and VAS scores and pain duration was calculated for the CTN group using SPSS 26.0 software. It revealed that in the CTN group, deci_value was positively correlated with the VAS scores in PCA( $r=0.42, \mathrm{P}=0.041$, twotailed). (Fig 3) While there was no difference between deci_value and VAS scores in F-score, T-test, Spearman ( $r=0.39, p=0.06 / r=0.36, p=0.09 / r=0.37, p=0.08$ respectively). We also did not find significant correlations between the pain duration for CTN patients and deci_value among the four methods of feature dimension reduction $(P>0.05)$. (Table 3 )

Table 3 Correlation between VAS scores and the deci_value in CTN patients with the different methods of feature dimension reduction. 


\begin{tabular}{|lllll|}
\hline & Spearman & T-test & F-score & PCA \\
\hline VAS & & & & \\
\hline$r$ & 0.37 & 0.36 & 0.39 & 0.42 \\
\hline$p$ & 0.08 & 0.09 & 0.06 & $0.04^{*}$ \\
\hline Duration & & & & \\
\hline$r$ & 0.39 & 0.19 & 0.22 & -0.04 \\
\hline$p$ & 0.06 & 0.37 & 0.31 & 0.86 \\
\hline
\end{tabular}

\section{Features Survive Every LOOCV}

Fig. 4 shows the result of features that survived in each iteration were concentrated in the region of the anterior cingulate cortex(ACC), superior frontal gyrus(SFG), and cerebellum inferior(Cl). As for F-score, ACC_L, SFG_R, and bilateral Cl were survived, and ACC_L, SFG_R, and CI_L was survived in the Spearman and T-test, which also returned a mean weight of these features to reflect its importance in the classification. (Table 4)

Table 4 Survive features mean weight in every LOOCV

\begin{tabular}{lllll} 
& SFG_R & ACC_L & Cl_L & Cl_R \\
\hline Spearman & -2.1626 & -2.6989 & 5.2527 & - \\
\hline T-test & -1.3654 & -3.4272 & 4.8472 & - \\
\hline F-score & -1.3638 & -3.2019 & 4.5580 & 1.2432
\end{tabular}

ACC: anterior cingulate cortex, SFG: superior frontal gyrus, Cl: cerebellum inferior, R:right, L: left

\section{Features Survive Every LOOCV}

The survival features were mainly concentrated in the region of ACC, SFG, Cl. The classification was driven by lower GMV of ACC_L, SFG_R and by higher GMV of Cl in the TN participants. All the features selected for SVM classification were significantly different at the group level in previous studies ${ }^{[20-22]}$. Besides, the contribution of these features to prediction is known from the feature weights derived from the linear model. the absolute value of feature weight can quantify the contribution of features to classification or prediction ${ }^{[26]}$. 
Anterior cingulate cortex (ACC) is an important part of the limbic system, also it is an important part of the pain matrix and highlighting network. It has a wide range of fiber connections with many other brain regions of the brain and is an important hub of the pain pathway. According to the analysis of painrelated neuroimaging studies, it is found that brain areas such as ACC and frontal cortex are continuously activated by continuous pain stimulation, which is considered to play an important role in sensory discrimination, cognition, and emotion of pain ${ }^{[27]}$. Therefore, ACC is considered to be the most important brain area for the development of chronic pain ${ }^{[28]}$. This indicates that the GMV in ACC can objectively reflect the degree of chronic pain to some extent ${ }^{[29]}$.

The frontal lobe belongs to the advanced information processing area, which does not directly receive the projection of the spinothalamic tract but can integrate the primary pain information into pain perception. Most chronic pain can affect the activity of these brain regions and even the structure of gray matter, and affect some advanced brain functions ${ }^{[30,31]}$.

The effects of pain on the cerebellum mainly include pain regulation, emotional processing, and sensorimotor processing ${ }^{[30,31]}$. In addition, the cerebellum can receive fiber projections from the spinal cord and trigeminal nerve, and long-term intense pain stimulation can lead to structural changes in its local gray matter ${ }^{[33]}$. To sum up, there are significant abnormalities in $\mathrm{GMV}$ in $\mathrm{ACC}$ and $\mathrm{Cl}$ involved in pain transmission, as well as in the SFG involved in pain perception and integration in patients with CTN. The interaction between these brain structures and chronic pain in TN may be one of the important mechanisms for the occurrence and development of TN.

\section{Discussion}

\section{Application of SVM algorithm}

SVM is a kind of generalized linear classifier that classifies the data according to the supervised learning method, and its deci_value is to solve the maximum margin hyperplane for the learning samples ${ }^{[14]}$. Compared with other algorithms, the advantage of the SVM algorithm ${ }^{[14,19]}$ is that it can be used for both classification and regression analysis; in the process of analysis, the SVM algorithm is to find the maximum interval hyperplane that can be classified, so that it has a certain tolerance and is not easy to over-fit; SVM is also very suitable for data with small sample size but high feature dimension of a single sample, which is a typical manifestation of fMRI data.

\section{SVM classification}

This study has focused on the analysis of gray matter imaging data in CTN patients to determine whether the neuroimaging derived structural patterns are sufficiently robust to be distinguished from HC at an individual level using an SVM-based algorithm. Our model applying PCA methods successfully identified CTN from $\mathrm{HC}$ with an accuracy of $85 \%$, with $91.67 \%$ sensitivity, and $86.36 \%$ specificity, and 
highlighted that the model used the top 18 PC to explain more than $90 \%$ of the original features in this differentiation. This is an important finding since up till now, the neuroimaging of TN pain has focused primarily on group distinctions ${ }^{[20-22]}$ and not on individual level characterization.

\section{Limitations}

A limitation of this study is a relatively small sample size. Nonetheless, our successful application of GMV in the classification of CTN and HC demonstrated its feasibility as features for the prediction of CTN. Our study focuses on GMV yet there are well-known other indicators abnormal at the group level, such as regional homogeneity $(\mathrm{ReHo})^{[34]}$ and fractional anisotropy $(\mathrm{FA})^{[35,36]}$ and so on. Future work could combine these information with additional types of neuroimaging data in order to examine whether this leads to higher levels of diagnostic accuracy and make the pattern more detailed comprehensive in the future.

\section{Conclusions}

In summary, the present study revealed spatially distributed subtle differential patterns of GM abnormalities in CTN patients, and indicated that these abnormalities allow accurate discrimination between CTN patients and $\mathrm{HC}$ at an individual level. And this study not only highlights the high accuracy of the PCA method, but also the role of ACC, SFG, and Cl for classification.

\section{Abbreviations}

CTN:classification between classic trigeminal neuralgia; TN:trigeminal neuralgia; HC:healthy controls; GMV:gray matter volume; GM:gray matter; WM:white matter; PCA:principal component analysis; SVM:support vector machine; ACC:anterior cingulate cortex; SFG: superior frontal gyrus; Cl:cerebellum inferior; MRI:magnetic resonance imaging; VBM:voxel-based morphometry; ROl:regions of interest; CSF:cerebrospinal fluid; MNI:Montreal Neurological Institute; FWHM:full-width at half maximum; LOOCV: leaving-one-out cross validation; AUC:area under the curve; ROC:Receiver Operating Characteristic; ReHo:regional homogeneity; FA:fractional anisotropy

\section{Declarations}

\section{Ethics approval and consent to participate}

The present study was approved by the Medical Research Ethics Committee of The First Affiliated Hospital of Nanchang University.Written informed consent was obtained from individual or guardian participants.

\section{Consent for publication}




\section{Competing interest:}

The authors declare that they have no competing interests

\section{Funding}

Thanks to the Natural Science Key Project of Jiangxi Province (20181BAB205063) for funding.

\section{Authors' contributions}

Xinji Peng: conception and design of the study, data interpretation, frst manuscript drafting. You Chen: subjects' recruitment, data acquisition. Guoshu Zhao, Huifang Du, Ziyi Hu: manuscript review. Laichang He: Supervision, review \& editing. All authors read and approved the fnal manuscript

\section{Acknowledgments}

Our sincere gratitude goes to all participants involved in volunteering their time to be part of this study. We also appreciated Dr. Zhou (Department of Radiology, the First Affiliated Hospital of Nanchang University) for revise the manuscript.

\section{References}

1. Maarbjerg S, Di Stefano G, Bendtsen L, et al. Trigeminal neuralgia - diagnosis and treatment[J]. Cephalalgia. 2017;37(7):648-57.

2. Cheshire WP. Trigeminal neuralgia: for one nerve a multitude of treatments[J]. Expert Rev Neurother. 2007;7(11):1565-79.

3. Tolle T, Dukes E, Sadosky A. Patient burden of trigeminal neuralgia: results from a cross-sectional survey of health state impairment and treatment patterns in six European countries[J]. Pain Pract. 2006;6(3):153-60.

4. Cruccu G, Finnerup NB, Jensen TS, et al. Trigeminal neuralgia: New classification and diagnostic grading for practice and research[J]. Neurology. 2016;87(2):220-8.

5. Li M, Yan J, Li S, et al. Reduced volume of gray matter in patients with trigeminal neuralgia[J]. Brain Imaging Behav. 2017;11(2):486-92.

6. Wang Y, Cao DY, Remeniuk B, et al. Altered brain structure and function associated with sensory and affective components of classic trigeminal neuralgia[J]. Pain. 2017;158(8):1561-70. 
7. Wang Y, Zhang Y, Zhang J, et al. Structural and functional abnormalities of the insular cortex in trigeminal neuralgia: a multimodal magnetic resonance imaging analysis[J]. Pain. 2018;159(3):50714.

8. Lindquist MA, Krishnan A, Lopez-Sola M, et al. Group-regularized individual prediction: theory and application to pain[J]. Neuroimage. 2017;145(Pt B):274-87.

9. Brodersen $\mathrm{KH}$, Wiech $\mathrm{K}$, Lomakina El, et al. Decoding the perception of pain from fMRI using multivariate pattern analysis[J]. Neuroimage. 2012;63(3):1162-70.

10. Gaonkar B, Davatzikos C. Analytic estimation of statistical significance maps for support vector machine based multi-variate image analysis and classification[J]. Neuroimage. 2013;78:270-83.

11. Bagarinao E, Johnson KA, Martucci KT, et al. Preliminary structural MRI based brain classification of chronic pelvic pain: A MAPP network study[J]. Pain. 2014;155(12):2502-9.

12. Ung $\mathrm{H}$, Brown JE, Johnson KA, et al. Multivariate classification of structural MRI data detects chronic low back pain[J]. Cereb Cortex. 2014;24(4):1037-44.

13. Jes Olesen L, Bendtsen, et al. Headache Classification Committee of the International Headache Society (IHS) The International Classification of Headache Disorders, 3rd edition[J] Cephalalgia, 2018,38(1):1-211.

14. Zhou Zhihua. Machine Learning[M]. Beijing: Tsinghua University Press; 2016.

15. Zhong J, Chen DQ, Nantes JC, et al. Combined structural and functional patterns discriminating upper limb motor disability in multiple sclerosis using multivariate approaches[J]. Brain Imaging Behav. 2017;11(3):754-68.

16. Mourao-Miranda J, Reinders AA, Rocha-Rego V, et al. Individualized prediction of illness course at the first psychotic episode: a support vector machine MRI study[J]. Psychol Med. 2012;42(5):1037-47.

17. Li F, Huang $X$, Tang W, et al. Multivariate pattern analysis of DTI reveals differential white matter in individuals with obsessive-compulsive disorder[J]. Hum Brain Mapp. 2014;35(6):2643-51.

18. Ecker C, Marquand A, Mourao-Miranda J, et al. Describing the brain in autism in five dimensionsmagnetic resonance imaging-assisted diagnosis of autism spectrum disorder using a multiparameter classification approach[J]. J Neurosci. 2010;30(32):10612-23.

19. Li Hang. Statistical Learning Method[M]. Beijing: Tsinghua University Press; 2019.

20. DeSouza DD, Hodaie M, Davis KD. Abnormal trigeminal nerve microstructure and brain white matter in idiopathic trigeminal neuralgia[J]. Pain. 2014;155(1):37-44.

21. Desouza DD, Moayedi M, Chen DQ, et al. Sensorimotor and Pain Modulation Brain Abnormalities in Trigeminal Neuralgia: A Paroxysmal, Sensory-Triggered Neuropathic Pain[J]. PLoS One. 2013;8(6):e66340.

22. Obermann $M$, Rodriguez-Raecke $R$, Naegel $S$, et al. Gray matter volume reduction reflects chronic pain in trigeminal neuralgia[J]. Neuroimage. 2013;74:352-8.

23. Schmidt-Wilcke T, Hierlmeier S, Leinisch E. Altered regional brain morphology in patients with chronic facial pain[J]. Headache. 2010;50(8):1278-85. 
24. Wang $Y$, Cao DY, Remeniuk $B$, et al. Altered brain structure and function associated with sensory and affective components of classic trigeminal neuralgia[J]. Pain. 2017;158(8):1561-70.

25. Obermann M, Rodriguez-Raecke R, Naegel $S$, et al. Gray matter volume reduction reflects chronic pain in trigeminal neuralgia[J]. Neuroimage. 2013;74:352-8.

26. Erus G, Battapady H, Satterthwaite TD, et al. Imaging patterns of brain development and their relationship to cognition[J]. Cereb Cortex. 2015;25(6):1676-84.

27. Morton DL, Sandhu JS, Jones AK. Brain imaging of pain: state of the art[J]. J Pain Res. 2016;9:61324.

28. Obermann M, Rodriguez-Raecke R, Naegel $S$, et al. Gray matter volume reduction reflects chronic pain in trigeminal neuralgia[J]. Neuroimage. 2013;74:352-8.

29. Yuan J, Cao S, Huang Y, et al. Altered Spontaneous Brain Activity in Patients With Idiopathic Trigeminal Neuralgia: A Resting-state Functional MRI Study[J]. Clin J Pain. 2018;34(7):600-9.

30. Mansour ZM, Lepping RJ, Honea RA, et al. Structural Brain Imaging in People With Low Back Pain[J]. Spine (Phila Pa 1976). 2017;42(10):726-32.

31. Del CA, Ferracuti S, Rapinesi C, et al. Pain perception and hypnosis: findings from recent functional neuroimaging studies[J]. Int J Clin Exp Hypn. 2015;63(2):144-70.

32. Moulton EA, Schmahmann JD, Becerra L, et al. The cerebellum and pain: passive integrator or active participator?[J]. Brain Res Rev. 2010;65(1):14-27.

33. Shi H, Yuan C, Dai Z, et al. Gray matter abnormalities associated with fibromyalgia: A meta-analysis of voxel-based morphometric studies[J]. Semin Arthritis Rheum. 2016;46(3):330-7.

34. Wang Y, Zhang X, Guan Q, et al. Altered regional homogeneity of spontaneous brain activity in idiopathic trigeminal neuralgia[J]. Neuropsychiatr Dis Treat. 2015;11:2659-66.

35. DeSouza DD, Hodaie M, Davis KD. Abnormal trigeminal nerve microstructure and brain white matter in idiopathic trigeminal neuralgia[J]. Pain. 2014;155(1):37-44.

36. Szabo N, Kincses ZT, Pardutz A, et al. White matter microstructural alterations in migraine: a diffusion-weighted MRI study[J]. Pain. 2012;153(3):651-6.

\section{Figures}




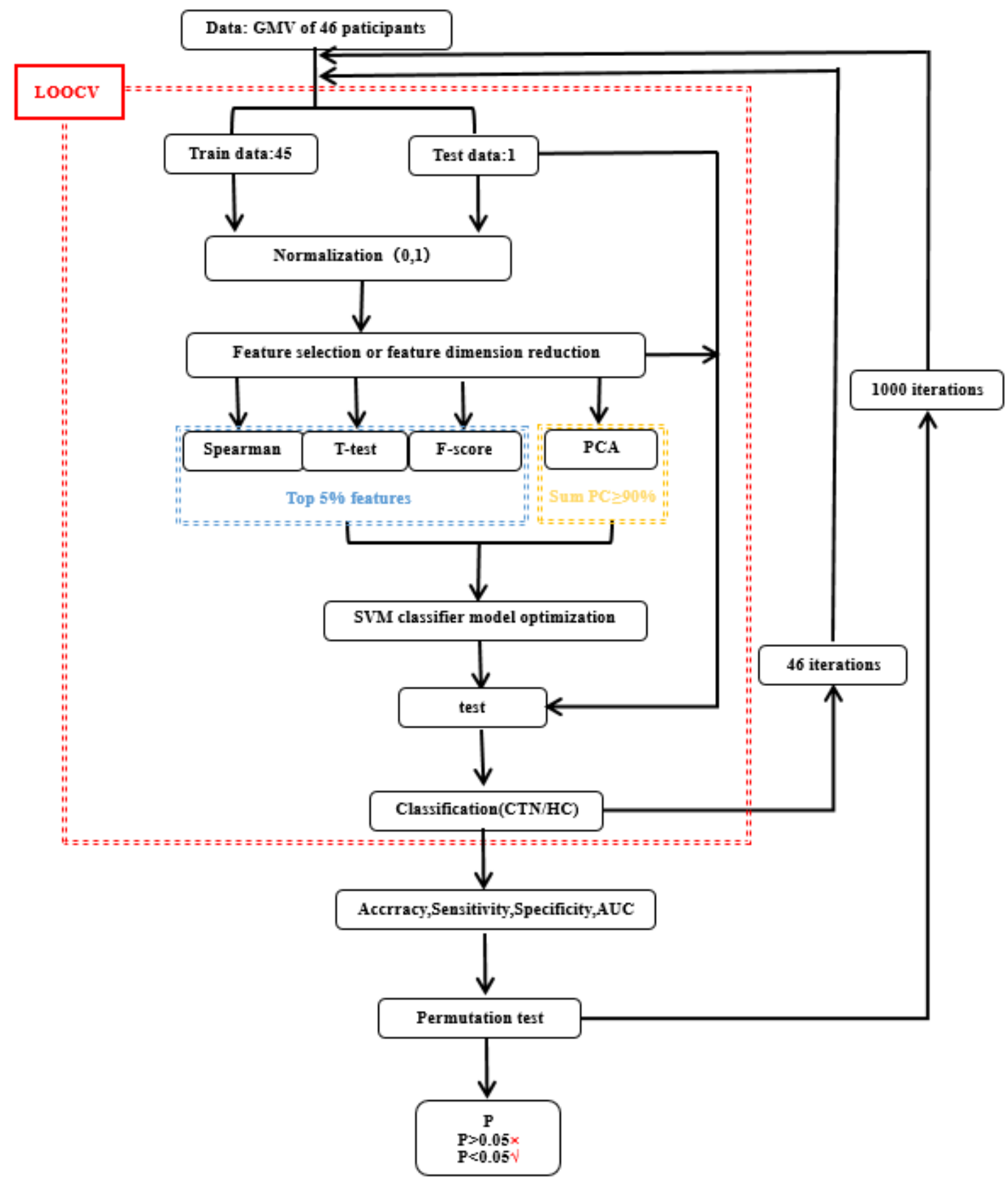

Figure 1

SVM classification flow chart. 


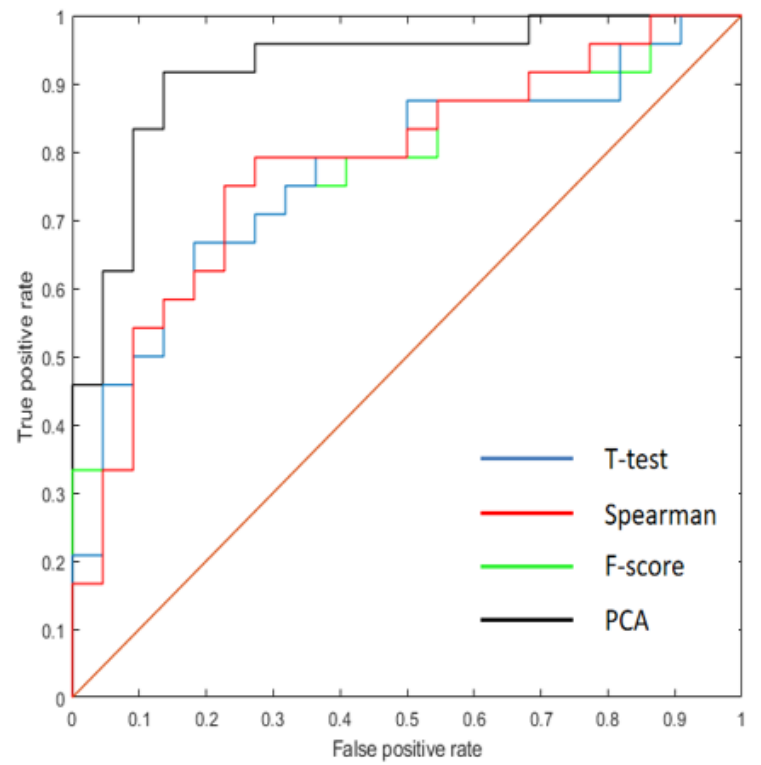

A

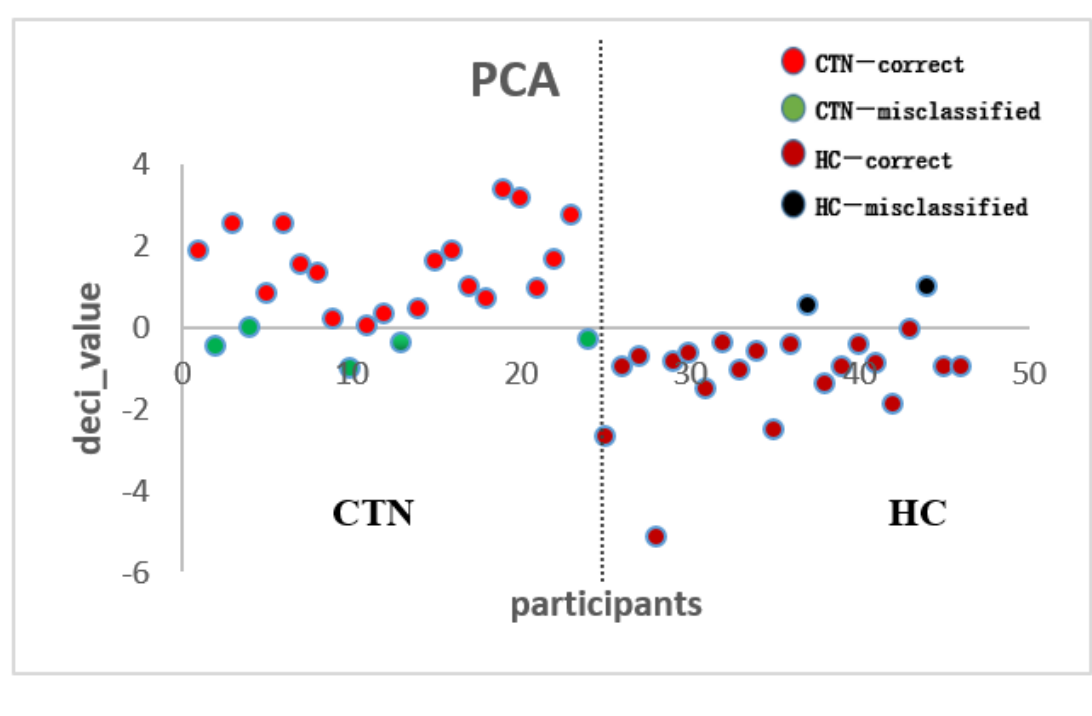

B

Figure 2

A The ROC curve of different methods of feature dimension reduction for classification between CTN and $\mathrm{HC}$ based on GMV. Figure 2B The distance from the sample to the optimal hyperplane(deci_value $=0$ ) for individual CTN participants and individual HC. SVM prediction: positive distance is classified as CTN, while negative distance as $\mathrm{HC}$.

\section{PCA}

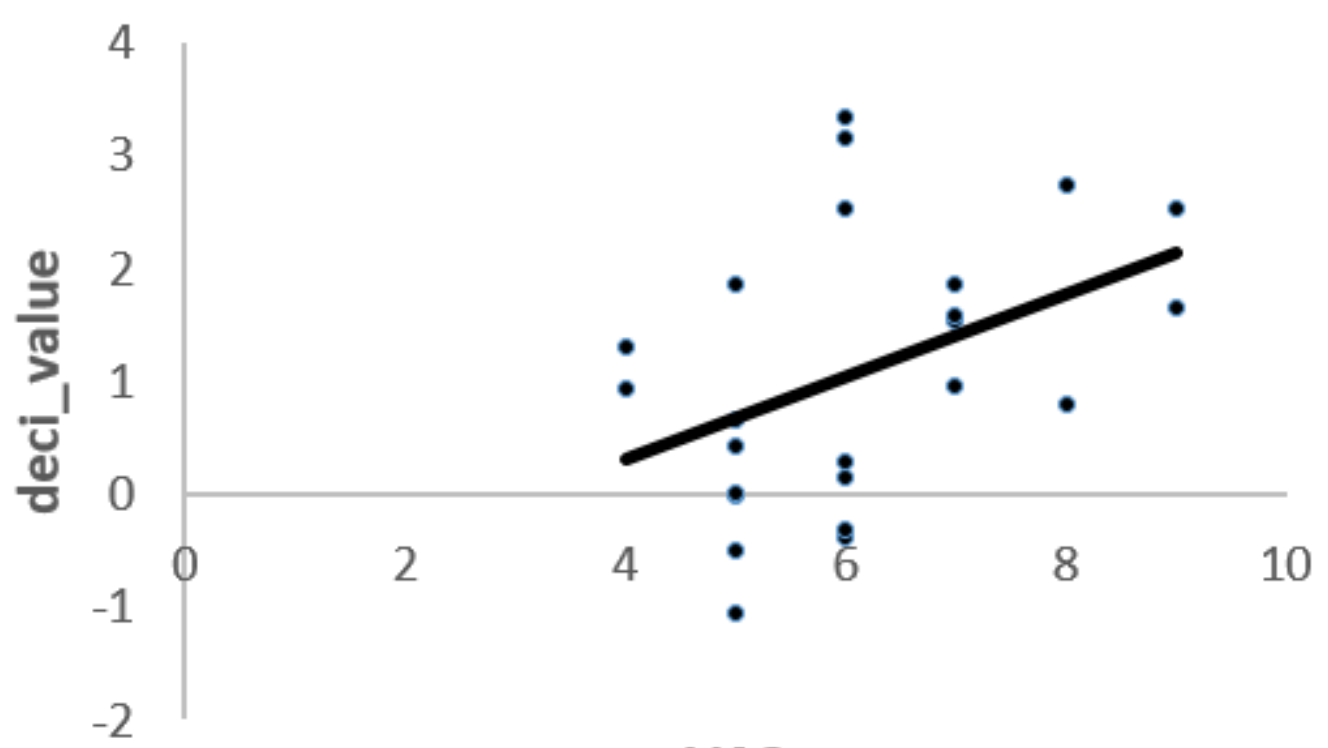

VAS

Figure 3

Correlation between VAS scores and the deci_value in CTN patients with the methods of PCA. 
$\mathbf{R}$

\section{Superior Frontal Gyrus}

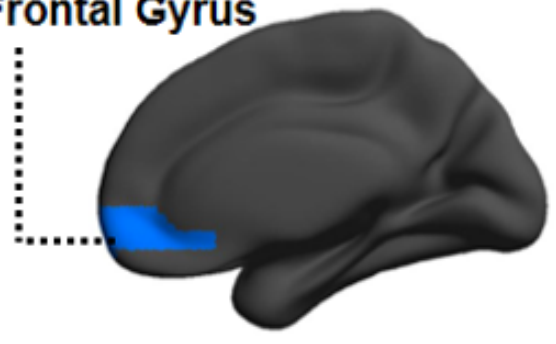

$\mathbf{L}$

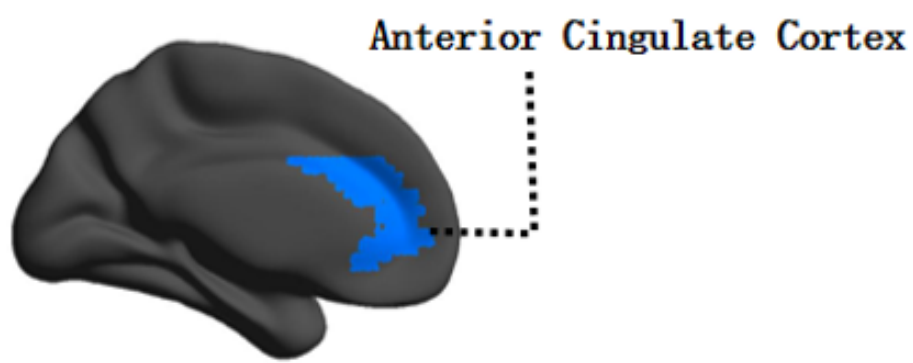

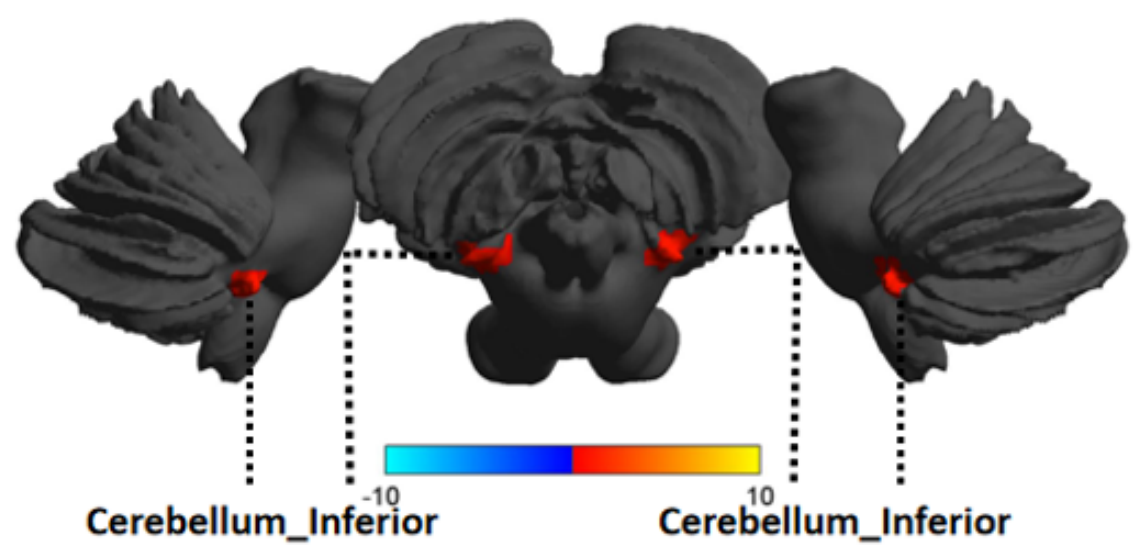

Figure 4

Features survive every LOOCV, the blue region indicates that CTN patients have higher GMV in a particular region than $\mathrm{HC}$, while the red region indicates the opposite. 